believed that lessons could be learned from the mistakes of the Ottomans, as well as their successes, to improve the British monarchal administration. Butler's analysis helps us view this work and its popularity in the context of the English Restoration and Britain's struggle to create internal stability and strengthen its economic position in the global market.

ARAZOO FEROZAN

McMaster University

\title{
Servetus, Michel.
}

Apologie contre Leonhart Fuchs. Édition et traduction de Jean Dupèbe.

Cahiers d'Humanisme et Renaissance, 143. Genève : Droz, 2017. cxlviii, 52 p. ISBN 978-2-600-05836-0 (broché) 42 CHF.

Commençons d'emblée par saluer la publication d'un texte de Michel Servet, souvent mieux connu pour sa fin tragique, sur le bûcher, le 27 octobre 1553 à Genève, que pour son œuvre. Mieux encore, l'édition et traduction de l'Apologie contre Leonhart Fuchs par les soins de Jean Dupèbe permet non seulement de lire ce texte bref, mais surtout de le situer, d'en comprendre la teneur et les enjeux. Exemplaire par sa méthode, par la minutie des recherches et par la concision de sa rédaction, la longue introduction de Jean Dupèbe, de 145 pages, est en effet un modèle d'explication de texte, non pas comme une lectio qui suivrait ligne à ligne ou mot à mot son objet mais comme la peinture d'une fresque qui donne au détail, en l'occurrence l'opuscule de Servet, de moins de 15 pages, une place dans l'histoire littéraire, l'histoire des idées et, tout simplement, l'histoire. Le sage de l'apologue chinois pouvait lire l'histoire du monde dans un grain de riz : Jean Dupèbe fait vivre autour de l'opuscule l'univers bouillonnant, contradictoire, changeant, de la première moitié du $\mathrm{XVI}^{\mathrm{e}}$ siècle sans recourir à la conjecture ni à l'emphase : en dévidant les fils de l'érudition attachés aux dates, lieux, personnes et œuvres.

Imprimée et publiée à Lyon en 1536, l'Apologie contre Leonhart Fuchs est à la fois une œuvre de circonstance, la première publication en faveur de Symphorien Champier de Michel Servet, et l'épitome d'une carrière intellectuelle nourrie de conflits, d'engagements et de mystères. Sous le signe d'une controverse entre personnes - le maitre de Servet, médecin français et 
catholique, Symphorien Champier et le médecin allemand luthérien Leonhart Fuchs -, l'opuscule dévoile en effet la complexité éthique et l'importance des loyautés personnelles et religieuses dans la France de François I ${ }^{\text {er }}$.

L'introduction de Jean Dupèbe part du texte pour en définir, d'abord, la nature : l'apologie humaniste, et la genèse, dans le parcours déjà mouvementé de l'étudiant espagnol, poursuivi par la Faculté de théologie pour ses positions sur la Trinité publiées en 1532 et 1533 . Humaniste par la connaissance de la philologie et la rigueur toute grammairienne de ses lectures, Michel Servet change de nom et de milieu pour s'attacher à un maître, Symphorien Champier, qui lui ouvrira les portes de l'université. Le portrait de Symphorien Champier occupe la plus grande partie de l'introduction : professeur, humaniste, médecin, plagiaire, homme de pouvoir, catholique engagé, figure symbolique pour la génération montante des années 1530, le maître que se choisit Servet est au centre d'un réseau intellectuel, religieux et professionnel. Les pages que Jean Dupèbe lui consacre n'ont rien d'une digression; elles confèrent à l'intervention de Servet dans un conflit qui le dépasse une dimension que le lecteur ne saurait saisir sans l'expertise et l'érudition que la préface lui transmet. En restituant, derrière chaque nom propre, un réseau, un parcours et des positions publiques, Jean Dupèbe décrypte ce qui aurait pu passer pour l'opportunisme d'un jeune auteur aussi désordonné qu’outrecuidant. Surtout, il dépeint, de manière à la fois précise et générale, ce que représente et implique l'engagement religieux d'un intellectuel dans la première moitié du siècle.

La dernière partie de l'introduction ajoute encore à la palette de cette fresque. En présentant le second maitre de Servet, le savant dominicain Pagnini, Jean Dupèbe entraîne le lecteur dans les méandres européens de la querelle religieuse, dont les contours n'épousent pas exactement ceux des querelles humanistes ou savantes. L'entrée en controverse de l'étudiant se continue ainsi par les reniements, en 1537, avec La théorie générale des sirops publiée chez Simon de Colines à Paris, de l'Apologie de 1536.

La plaquette seule, même accompagnée de son excellent appareil de notes et de sa traduction sans faille, eût failli à retenir l'attention des lecteurs du XXI e siècle, peu au fait des intrigues, allusions et sujets brûlants de 1536 . L'introduction magistrale de Jean Dupèbe, qui se lit comme un roman, la place ainsi dans une histoire palpitante, celle de la modernité de la Renaissance, une modernité qui se cherche, se retourne, se dédit et, finalement, tombe sous les coups des censures et des tribunaux religieux. Grâce à la préparation de la 
lecture, qui donne littéralement accès à la signification perdue des références et enjeux, la plaquette aux sujets multiples - La Foi et les œuvres, la Scammonée, le Mal français en sont les têtes de chapitres - , aux accents polémiques et à la lourde érudition, s'inscrit dans le foisonnement des interrogations des années 1530 tout autant qu'elle éclaire les obscures contradictions de son époque.

Ajoutons qu'une note sur les poids et mesures, ainsi qu'un index des noms propres pallient les défauts de mémoire ou de langue des lecteurs. S’il l'on devait alors regretter une absence, dans ce volume si plein, ce serait celle d'une table des matières qui aurait détaillé les parties de l'introduction, signalée comme un bloc monolithique au sommaire.

Cette édition fait déjà référence, non pas tant pour l'édition critique d'une plaquette somme toute étrange et reniée sitôt publiée, que pour la magistrale peinture par l'éditeur et traducteur, des déchirements humanistes de la première moitié du XVI ${ }^{e}$ siècle.

HÉLÈNE CAZES

Université de Victoria

\section{Sforza, Ippolita Maria.}

Duchess and Hostage in Renaissance Naples: Letters and Orations. Ed. and trans. Diana Robin and Lynn Lara Westwater.

The Other Voice in Early Modern Europe: The Toronto Series 55 / Medieval and Renaissance Texts and Studies 518. Toronto: Iter Press/Tempe: Arizona Center for Medieval and Renaissance Studies, 2017. Pp. xvi, 229. ISBN 978-086698-574-1 (paperback) US\$39.95.

Ippolita Maria Sforza (1445-48) was a tremendously interesting Renaissance woman writer whose rich political life, as illustrated by her letters and Latin orations, deserves far more attention than it has received. The daughter of the beleaguered but astute Duke of Milan, Francesco Sforza (1401-66), and of Bianca Maria Visconti (1425-68), Ippolita was at front and centre of the complex politics of Renaissance Italy. Married at the age of twenty to Alfonso II, Duke of Calabria (1448-95), King of Naples (r. 1494-95), not only was she duchess and consort, she was also an astute diplomat and politician. Her importance, however, has been underplayed and English-language scholarship 